\title{
Kikiskawâwasow - prenatal healthcare provider perceptions of effective care for First Nations women: an ethnographic community-based participatory research study
}

Richard T. Oster ${ }^{1 *}$, Grant Bruno ${ }^{2,3}$, Margaret Montour ${ }^{3}$, Matilda Roasting ${ }^{4}$, Rick Lightning ${ }^{5}$, Patricia Rain ${ }^{3}$, Bonny Graham ${ }^{6}$, Maria J. Mayan ${ }^{7}$ Ellen L. Toth ${ }^{1}$ and Rhonda C. Bell ${ }^{2}$

\begin{abstract}
Background: Pregnant Indigenous women suffer a disproportionate burden of risk and adverse outcomes relative to non-Indigenous women. Although there has been a call for improved prenatal care, examples are scarce. Therefore, we explored the characteristics of effective care with First Nations women from the perspective of prenatal healthcare providers (HCPs).

Methods: We conducted an ethnographic community-based participatory research study in collaboration with a large Cree First Nations community in Alberta, Canada. We carried out semi-structured interviews with 12 prenatal healthcare providers (HCPs) that were recorded, transcribed, and subjected to qualitative content analysis.

Results: According to the participants, relationships and trust, cultural understanding, and context-specific care were key features of effective prenatal care and challenge the typical healthcare model. HCPs that are able to foster sincere, non-judgmental, and enjoyable interactions with patients may be more effective in treating pregnant First Nations women, and better able to express empathy and understanding. Ongoing HCP cultural understanding specific to the community served is crucial to trusting relationships, and arises from real experiences and learning from patients over and above relying only on formal cultural sensitivity training. Consequently, HCPs report being better able to adapt a more flexible, all-inclusive, and accessible approach that meets specific needs of patients.

Conclusions: Aligned with the recommendations of the Truth and Reconciliation Commission of Canada, improving prenatal care for First Nations women needs to allow for genuine relationship building with patients, with enhanced and authentic cultural understanding by HCPs, and care approaches tailored to women's needs, culture, and context.
\end{abstract}

Keywords: Indigenous population, Qualitative research, Prenatal care, Community-based participatory research, Health care providers

\footnotetext{
* Correspondence: roster@ualberta.ca

${ }^{1}$ Department of Medicine, Research Transition Facility, University of Alberta,

Edmonton, AB T6G 2V2, Canada

Full list of author information is available at the end of the article
} 


\section{Background}

In the Cree language, kikiskawâwasow means "she is with child". Pregnancy is a crucial period in the health trajectory of a family. In addition to acute impacts, the fetal circumstances in utero substantially affect future risk of developmental difficulties/challenges and adult diseases [1]. A growing body of perinatal research has detailed considerable global health disparities experienced by Indigenous women and their infants compared to non-Indigenous populations [2]. Similar disparities have been shown among Canada's most populous Indigenous group ${ }^{1}$, the First Nations, in Alberta [3, 4]. Moreover, among First Nations women fertility/birth rates are significantly higher and inadequate prenatal care occurs more often than in non-First Nations women $[5,6]$. Specifically, pregnant First Nations women in British Columbia (Canada) were less likely to have early ultrasonography, to have at least four antenatal care visits, and to undergo labour induction after prolonged pre-labor rupture of membranes or at postdates gestation compared to non-First Nations women [6]. Improved care and a greater understanding of how to effectively work in this context is needed.

Frequent dissatisfaction with prenatal healthcare providers (HCPs) has been shown in qualitative work with Indigenous women [7-11]. On the other hand, from the perspective of prenatal HCPs, lack of time and systemlevel resources are often described as barriers to appropriate care for Indigenous women [12, 13]. This report is part of a community-based participatory research (CBPR) project in collaboration with a large Cree First Nations community in Alberta. It builds from an ongoing community engagement process that has taken place over several years. Our objective was to explore the characteristics of effective care with First Nations women from the perspective of HCPs that service a large First Nations community in Alberta. For our purposes, effective care essentially referred to care that contributed to healthier pregnancies and better prenatal outcomes. This work is one piece of the overarching ENRICH study (www.enrich.ales.ualberta.ca), which aims to improve maternal health in pregnancy and postpartum within Alberta.

\section{Methods}

\section{Study design and setting}

This study was carried out in collaboration with the Cree community of Maskwacis, Alberta (collectively made up of Samson, Louis Bull, Montana, and Ermineskin First Nations bands; includes Pigeon Lake reserve), with which we have pre-existing research relationships. Our approach was informed by both ethnography and CBPR. The foundation of CBPR is true partnerships between the researcher and community members to resolve significant social and/or health problems [14]. CBPR considers community members as experts on their own experience, carrying knowledge and skills that are valuable to the research process [15]. Ethnography is a qualitative method that is increasingly being adapted in healthcare settings to view and understand perceptions, beliefs, shared meanings, values, and/or practices in the context in which they occur $[16,17]$.

Our community partners voiced a need to improve the pregnancy-related health of women from the community, and desired a CBPR collaboration. After an initial year and a half of engagement activities within the community by Oster (e.g. developing strong relationships and friendships, volunteering at the health centres, attending community events, participating in sub-committee meetings, taking part in ceremonies and cultural events, assisting with ongoing research projects in the community, making presentations when asked, etc.) a research partnership was formed through the development of a Community Advisory Committee (CAC). Childbearing women from Maskwacis were involved in the engagement activities to help better understand the community context and to inform the CAC meetings. The CAC acted as our main community collaborator and consisted of a fluid group of community members, Elders, and staff from the community health/social services departments. Through regular collaborative meetings, the CAC provided guidance and advice to all involved and devoted time to jointly design the research protocol, contribute to the interpretation of the data, and approve all dissemination pieces. Several research objectives were established, including understanding the perceptions of HCPs (current study), fathers (parallel study), as well as Elders and women of childbearing age (parallel study). The CAC partnership agreed ethnography would be the most appropriate approach to achieve the research objectives as it invokes a collaborative process that seeks to involve participants in the research process, and is both compatible with and complimentary to CBPR [16].

Maskwacis is a rural community (not remote) approximately $90 \mathrm{~km}$ south of Edmonton, Alberta, within the area of Treaty Six. The registered population of the four bands is 16,004. Pregnant women must travel to neighboring off-reserve towns and cities for prenatal care, with the majority receiving care at a single Primary Care Network associated with delivery at a Hospital that is approximately $15 \mathrm{~km}$ from the community town site. However, some pregnancy-related care and most postnatal care is delivered within the community (e.g. prenatal classes, diabetes clinic which sees women with diabetes complicating pregnancy, home care team, immunizations and postpartum care, etc.) and in other surrounding communities. 


\section{Ethics}

Ethical approval by the University of Alberta Research Ethics Board was obtained. Community approval from key stakeholders and Elders was also obtained. Community members were involved at all stages of this project, with the aim of shared power, equitable resourcing and mutual understanding. The CAC also provided ethical assistance and ensured the research progressed in a culturally appropriate manner. A research agreement was jointly developed with the CAC. Throughout the study there was capacity building within the community through the involvement of community members whenever possible (e.g. hiring of community members as research assistants, locally sourcing any research-related expenditures, etc.).

\section{Sample}

HCPs (nurses, physicians, dietitians, mental health therapists) within Maskwacis and neighboring nonIndigenous communities were recruited using purposeful sampling. We wanted to attain a sample that was proportionately representative of those providers who deliver prenatal care to women from the community. Midwifery is generally uncommon in the province of Alberta, and to our knowledge there are no midwives working in this community. We recruited HCPs that worked regularly with pregnant and postpartum women from Maskwacis and provided health-related or comprehensive care to women during the childbearing year. We sought to have a mixture of Indigenous and nonIndigenous participants, and on-reserve and off-reserve participants. All of the participants had been working with women from Maskwacis for a minimum of one year. An information letter detailing the study was reviewed with HCPs who agreed to participate, and written informed consent was provided prior to interviews.

\section{Data generation}

The data were generated from June to December 2015 via one-to-one semi-structured interviews at a mutually selected location. The CAC partnership agreed that representative voice of the participants was the aim of the data generation activities and felt participant observation (often a core data collection method of ethnography) may alter the participant's behavior. Since participant observation was also agreed to be too time consuming for the project goals, this approach was not utilized. The interviews were conducted by Oster and Bruno in English, lasted approximately 45-60 $\mathrm{min}$, and were audio-recorded and transcribed verbatim. Bruno is of Indigenous descent. Interviews were conversational and open-ended questions were asked to prompt discussion (e.g. what kind of advice would you give another HCP that is working with pregnant Indigenous women?). It was our intent to highlight and understand positive practices and not to 'interrogate' HCPs or set an overcritical atmosphere for the interviews. The participants were encouraged to focus solely on prenatal care and their Indigenous pregnant patients.

\section{Data analysis}

Data were analyzed together by Oster and Bruno concurrently with data generation using qualitative content analysis [16] and Atlas.ti qualitative computer software. Briefly, transcripts were read and re-read, and coded to determine persistent concepts that were grouped in categories. The categories were again re-read (and reorganized if necessary), and were then described in-depth. The categories were considered together to determine if/ how they were related and to identify common themes in all of the data. Initial findings were discussed with the CAC and members of our research team, and feedback was sought from both of these groups. Feedback helped refine the categories and findings, and reduce redundancy. Data collection and analysis ceased upon data saturation, when no new information or insight emerged, and when the categories were well defined.

\section{Rigor}

Strength and rigor were achieved by regularly consulting the CAC and by abiding with the approaches described by Whittemore, Chase, \& Mandle [18] and Milne \& Oberle [19] to achieve authenticity, credibility, criticality and integrity of data collected. Oster and Bruno kept reflective personal journals throughout the entire research process to allow for reflection and critical appraisal of the information collected. Participants were provided with a draft of the findings and invited to provide feedback to enhance the trustworthiness and correctness of the data, eight of which did.

\section{Results}

A total of 12 participants were needed to reach data saturation. The average age of participants was 42 years. Six of the participants worked within the community and six worked off-reserve. Three of the 12 participants were Indigenous, which is representative of the HCPs available to pregnant women in this community and in the surrounding communities (Indigenous HCPs are the minority). Seven of the participants were nurses, two were physicians, two were dietitians, and one was a mental health therapist.

Three core categories that were highly interrelated and consonant with one another were identified from the analysis. The first category, Relationships and Trust, was foundational to the other two categories of Cultural Understanding and Context-Specific Care, and all three were identified as critical to effective prenatal care with 
First Nations women. Moving beyond the mainstream patient-provider relationship and the healthcare system as whole was also a central theme to the categories. An in-depth description of each category is presented below.

\section{Relationships and trust: "It's all about relationships"}

The participants felt strong relationships and trust with their Indigenous patients was crucial to effective prenatal care and ultimately leads to healthier pregnancies. Going beyond the usual patient-provider relationship by taking more time to get to know patients and including personal investments was necessary according to the participants to achieve genuine relationships and trust.

Building authentic relationships and trust with patients, particularly at the first visit, was uniformly identified by the participants as the basis of effective care. Participants felt such relationships contributed to the support networks of patients and helped reduce fear of the healthcare system that may have arisen from previous negative experiences, judgment, and discrimination. Building such relationships and trust was seen as making it more likely those patients would attend their appointments and be receptive to health messages/education. This was believed to result in better pregnancies: "We need to spend a lot more time building relationships if we are going to have better health outcomes." In many instances this involved getting to know patients over numerous clinic visits and having conversations about whatever that patient was dealing with that day, as one participant articulated:

I think the biggest thing is just trying to create that relationship with the moms. I know they are hesitant accessing health care. They may have had poor or bad experiences with other nurses... We don't know that patient's story. That's probably the first thing you could do is ask them. Not about why they're there at their appointment, but to try to talk to them and create a conversation. Ask them about who they are, about their family, and really try to get to know them... Really just being open to whatever they bring to us and talk about that first.

\section{Another participant went on:}

Never assume anything. Get to know that person. I always ask them to tell me their story, you know, what's going on. I don't go in with a paper and pen and start ticking off the baby's vital signs... I listen to their story and that's the first thing in gaining trust, because it might not be about having the baby or the physical labour. It might be that she had a fight with her boyfriend and she's really, really depressed and she could care less about the baby right now... The moms have my cell number, they can text me anytime.

Developing relationships of trust often entailed transcending the typical formal patient-HCP relationship, moving more towards personal investments and advocating for patients. This necessitated "treating them like a friend", "being respectful", "acknowledging their victories", "being open and honest", "getting to know the names and lives of these women", "asking open-ended questions", "being non-judgmental", "using a lot of humor", "empathy", "focusing on the positives", "sharing with them", "building rapport", "being a little bit vulnerable", "putting our egos aside", "being equal", and "just shutting up and listening a little bit", to name a few. One participant expressed the need for meaningful relationships beyond the conventional patient-HCP relationship:

It's about building one relationship at a time. It's about being a person. I don't think anybody thinks of me as a health professional... You have to be genuine. You can't be faking because that would be totally insincere and obviously wrong. But if you feel something is appropriate, say it. Like 'oh my gosh you are so beautiful.'... But I think sometimes professionals might be way too much to the book... You are not supposed to hug people apparently. Oh well, you know what? If they need a hug, they are getting one. And yeah, you are not supposed to use affectionate terms. You know what? Some of my clients are my 'dears'. Yep, some of them are my 'sweeties'.

Some participants described the need for changing system priorities to focus more on building relationships: "the organization likes to see numbers and they don't necessarily look at relationships, but until you actually build a relationship and you build that trust you're never going to get anywhere with that client." Establishing meaningful relationships and interaction with patients also required finding "the right staff". One participant explained: "They need to ask themselves 'why are they working with First Nations people?' If you don't know why, then maybe this isn't a good fit for you." Participants felt HCPs that are open, sincere, compassionate, willing to go "that extra mile", and able to understand the many issues their First Nations patients may be facing, are better suited to working with this population and are more likely to find such work enjoyable and an opportunity for mutual learning.

\section{Cultural understanding: "if you have never been out there, you really have no idea"}

According to the participants, Cultural Understanding specific to the community served is vital to trusting 
relationships and effective prenatal care, and entails not only an understanding of Indigenous culture but their histories and context as well. Enhanced cultural understanding was sought by all of the HCPs and was thought to be more impactful if it arose via real experiences and learning from patients rather than relying only on formal cultural sensitivity training alone.

Participants believed that better cultural understanding for HCPs was an important part of enhancing effective care of First Nations women and would further strengthen patient-provider relationships, lessen patient fear of the healthcare system, and provide a more welcoming environment for patients. The participants also felt enhanced cultural understanding would reduce provider frustrations, reduce provider stigmatization and discrimination of First Nations patients, further develop provider compassion and awareness, and encourage more appropriate care recommendations. Taken together, the participants believed this would decrease the number of missed appointments and improve care outcomes. One participant felt willingness to develop a deep cultural understanding should be "a prerequisite to anyone that's working with First Nations people on a regular basis."

All of the participants identified that they had an individual need for greater cultural understanding (even the Indigenous participants), and some longed to spend more time with Indigenous people and Elders to learn. The participants were interested and intrigued to know more about First Nations culture and history, and felt they would be more effective HCPs as a result. However, many described not knowing how or where to receive such knowledge or training, and were afraid of feeling "stupid or judged" or "offending someone" by not knowing the appropriate protocol to ask for cultural knowledge. The participants explained that although positive attempts have been made to improve the cultural sensitivity of the healthcare system in Alberta and in Canada, the system remains "nowhere near adequate." While receiving their health discipline training, the participants learned very little, if anything at all, about Indigenous culture or peoples. Moreover, most of the participants had never received any formal cultural sensitivity training despite the majority of their current work being focused on First Nations patients: "My entire training on cultural sensitivity was five minutes with a fellow from Health Canada. I have never had formal cultural sensitivity training." Another participant elaborated:

It wasn't enough. There were these bits and pieces here and there, kind of scattered all over. But no, not near enough. I truly believe that all health care providers, no matter what discipline they are in, need their own individual course that is a part of their program. A component that addresses Aboriginal health issues... The faster that happens, the more prepared health care providers will be to deal with them and the issues that we face.

Those participants that remember receiving some sort of training regarding cultural understanding, such as through manuals/documents or short presentations, described it as largely ineffective. However, as a result of having formed good, trusting relationships with their patients, some participants had learned from their patients and attained a greater awareness and appreciation of patients' context and culture: "I think it's important to ask, and lots of people are willing to tell you if you take the time to ask them." Also, authentic experiences with Indigenous people and Elders, ideally within communities, tended to have a much more profound effect on their cultural awareness and understanding:

We had an Elder come in and we did a big circle, and she told us her story. We were all crying and we had no idea. It was like we were hit by a truck...I also took a couple of courses with an Aboriginal teacher (that took place within) an old residential school... They can teach you things in books, but going into the school and walking past the morgue and hearing stories, that was a huge eye-opener of what people lived through and what lots of families lost. It definitely gave me a lot more compassion... Cultural sensitivity training in a classroom is great, but I think it's those relationships with community members, hearing someone tell their story is way more impactful.

Cultural understanding encompassed not just an appreciation and grasp of cultural practices, but also knowledge of the ongoing impacts of colonization, and the vulnerability and numerous challenges some patients may encounter: residential school legacy, ${ }^{2}$ racism, historical trauma, cultural loss, addictions, family violence, mental health issues, diabetes, crowded homes, food insecurity, poverty, poor support, limited opportunities, and lack of reliable transportation to name a few. Subsequently, HCPs were better able to understand why some First Nations women missed appointments (which was consistently cited as the most important barrier to prenatal care), as one participant described: "When you're dealing with all those social determinants of health, then attending appointments is not a priority. And I don't think it should be a priority either." Some participants felt it necessary to openly acknowledge the negative impact of colonization to encourage dialogue, stronger relationships, and cultural understanding. 
Context-specific care: "It is not one size fits all prenatal care" Context-specific care, although not always possible, was perceived by the participants as a key piece of effective prenatal care. They felt context-specific care requires both individual HCPs and the systems in which they work to adapt a more flexible, all-inclusive, and accessible approach that meets specific needs of their Indigenous female patients.

Providing more context-specific care at the individual HCP and health care system levels mitigated some of the barriers to care specific to First Nations women according to the participants. For instance, participants spoke of recent changes in their organization to provide a more open-door style of care, where pregnant women are able to receive care on a walk-in basis if they have not made an appointment, and the ability and willingness of staff to "stay open later just to accommodate certain people." In recognizing that missed appointments are common for a variety of reasons, participants expressed striving for "all-inclusive", "multi-disciplinary" and "single-session" care that offers "full service for prenatal care services" to make it easier for their patients. Participants also felt it is important to "meet them where they are at", as one participant explained: "Just work with the person in front of you and whatever is going on in their life. Working with people as if it might be the only time you see them."

Moving beyond current standard prenatal care was key. For example, one participant explained that she sometimes met with clients at alternative locations: "There is one girl that had just had a baby and wanted to go back to school. So I actually went to the school and I did the visitation there and it was really good." Other ideas for context-specific care that had either worked well in the past or were currently being advocated for included: Elder support/mentoring, lactation consultants, menopause programs, midwifery programs, and childcare (ideally accessible within the same facility). Further, improving the coordination of care between the many different clinics and hospitals in the area (both on and off-reserve) was viewed as vital to improving overall care. Participants regularly spoke of their patients struggling to navigate an often overwhelming and complex system.

Context-specific care with First Nations women called for a different 'bedside manner' than what many of the participants were used to and that often included genuine listening and counselling. Participants again spoke of the need for equality with patients rather than usual patient-provider relationships that often imply an imbalance of power. Accordingly, HCPs should "actually explain how things work instead of just telling people what to do." Further, one participant explained:
I'm not going to tell them like 'this is what you have to do', because that approach never works... You have to give control back to people, because when they feel like they're out of control already in their lives, its not going to be helpful when they come to our doors and they don't have any control at all.

Providing context-specific care, while identified as essential by many participants, was not always attainable within the healthcare system. Participants portrayed an often inflexible mainstream system built on structure, scheduled appointments, rules, policies, time management, order, etc. that did not necessarily meet the needs of First Nations women: "Our biggest thing is that we are kind of pigeon-holed into time." Another participant provided an additional poignant example: "In the past I have tried to advocate for clients getting their own homes. I got slapped on the wrist because we're not supposed to be political, when Aboriginal health is one of the most political things in Canada." Staff turnover was also described as a barrier to providing appropriate care and to women attending appointments, as the participants felt women need to see "familiar faces every time they come in." Participants expressed feeling "frustrated", "irritated", "guilty", "limited", "discouraged", and like their "hands are tied" when unable to provide care they felt essential for First Nations women due to system barriers. As was the case for building relationships and trust, providing context-specific care was limited not only by the 'system' but also by the individual as it requires the right staff that are willing to adapt their care approach.

\section{Discussion}

We sought to explore the characteristics of effective care with First Nations women from the perspective of HCPs in a large First Nations community in Alberta. Relationships and trust, cultural understanding, and contextspecific care were key features of effective prenatal care and challenge the usual healthcare model according to the participants.

There is no shortage of qualitative research with Indigenous women indicating relationships with prenatal HCPs are often poor, and women voice regularly experiencing impersonal and dismissive clinic visits as well as judgmental and seemingly untrustworthy HCPs [7-12]. Clearly there is room for improvement to develop positive and trusting relationships with HCPs, which Indigenous women desire [7,9]. In the group of HCPs we interviewed, they perceived that taking the time to invest in honest/trusting, heartfelt, and friendly interactions was crucial to successful relationships. This mirrors the perceptions of pregnant inner-city women living in a Canadian city (50\% of which were Indigenous), 
who described valuing $\mathrm{HCPs}$ that were trustworthy, non-judgmental, and took the time to make personal connections with patients [13]. Care approaches that are centered on building a respectful relationship with patients have been shown to lead to positive patient experiences, better clinical outcomes, patient compliance with care recommendations, and health-promoting behaviours in a wide range of populations [20-22].

Our findings add to a large and growing body of research calling for greater cultural understanding on the part of prenatal HCPs of Indigenous women [7-12, 23], including the recent World Health Organization recommendations on health promotion for maternal and newborn health [24]. A variety of terms are used in the literature, including cultural safety, awareness, competency, humility, appropriateness, and sensitivity, among others, but the message is the same: HCP cultural understanding of Indigenous women is lacking, and prenatal care and outcomes suffer as a result. HCPs, to be effective, must learn more about the extensive impacts of colonization and the resultant inequities in the social determinants of health, as well as the distinct cultural practices of the Indigenous group they are serving.

Excellent clinical practice guidelines exist aimed at improving the cultural understanding of prenatal HCPs (many of which recommend increasing the numbers of Indigenous HCPs), including recent Canadian consensus guidelines of the Society of Obstetricians and Gynaecologists [25]. However, our findings indicate that reading alone is likely not enough to achieve meaningful cultural understanding, and that it may be strengthened by shared experiences involving HCPs, patients and neighboring Indigenous communities in working together. Such collaborations would allow for mutual learning, real-life experiences, and relationship building, and could be potentially accomplished via attending cultural events and ceremonies, developing workshops led by Elders and/or community members, and involving Elders and/or community members in the clinic environment [26, 27]. More research is needed in Indigenous populations and to assess any impact of $\mathrm{HCP}$ cultural competence training on prenatal health status and pregnancy outcomes.

Context-specific care, as described by the participants in our study, requires flexibility and extra time and resources on the part of $\mathrm{HCPs}$ to establish relationships and rapport with patients and neighbouring clinics and hospitals, develop a strong cultural understanding, and tailor existing care models to better meet the needs of their patients. Our findings support an ethnography with First Nations women and University academics that found ineffective relationships with HCPs were often the result of structural limitations of the healthcare system [9]. Similarly, a recent qualitative description with prenatal HCPs treating pregnant inner-city women suggests healthcare system barriers (including HCP lack of time, shortage of HCPs) prevent appropriate prenatal care. The authors call for more accessible, convenient, and responsive prenatal care approaches [13]. Our findings also build upon qualitative research calling for equality and shared power in the patient-provider relationship to avoid fear-inducing, paternalistic and ineffective healthcare $[7,12]$. Solutions to improving care and strengthening HCP-patient relationships may lie in supporting efforts toward more localized and community-based care, such as Indigenous birthing centres and Indigenous midwifery $[10,25,28,29]$ of which the Inuulitsivik midwifery service and education program in the Inuit region of Quebec, Canada is an excellent example of [30]. Another example of such a program functioned briefly in the same town where some of our research was conducted [31], and ended because of lack of ongoing funding. This needs to change, funding for prenatal care is known to derive better pregnancy outcomes [32, 33].

Although Indigenous women often share a similar history of colonization and disparities in the social determinants of health, transferability of the results to other Indigenous populations and communities may be limited as communities are distinct in many ways. Some of the HCPs from surrounding non-Indigenous communities were unable to take part in interviews and we were unable to capture their experience and views. However, the fact that most of our participants were non-Indigenous and had a strong desire to be better HCPs, and hope that others would benefit from their mistakes and experiences, was an encouraging finding. Another limitation of this study is that we did not capture the perspectives of pregnant Indigenous women, only those of HCPs. Our CAC was adamant in allowing the HCPs to have their own voice. We are currently capturing the voice of childbearing women from the community in a parallel qualitative study.

\section{Conclusions}

Prenatal HCPs in Canada are likely to encounter Indigenous patients, and must have better knowledge and cultural expertise to improve this working relationship. Efforts are needed to repair and improve patientprovider relationships, focusing on investing time to develop enjoyable interactions, trust, mutual respect, and shared power with patients. Time invested up front should be foundational and not be seen as "extra". HCPs should acquire meaningful and positive cultural understanding of the Indigenous peoples they are working with. Reading seminal literature and/or completing online competency courses are important [32] but are not nearly enough. HCPs, their organizations, as well as 
institutions involved in training of HCPs could work to partner with Indigenous patients and communities to create opportunities for sharing real-life experiences and building positive, ongoing relationships that ultimately improve the integration of HCPs into communities. Any cultural understanding training needs to include components that are specific to the community since all are unique - reinforcing the need for active engagement, collaboration, and mutual learning. Current healthcare systems, clinics, and HCPs need to allow for more innovation, flexibility, and responsiveness in care approaches when working with Indigenous prenatal patients so that care is specific to their context. There is also a need for improved information sharing and coordination of care between hospitals and clinics that provide prenatal and obstetrical care to First Nations women.

Identifying practical ways to put the Calls to Action related to Health from the recent Truth and Reconciliation Commission [34] would go a long way in achieving the implications of our research (identified above) and in advancing the care and health of pregnant First Nations women. Specifically, this would include: acknowledging that current Aboriginal health is a direct result of colonization and of previous government policies (including residential schools); implementing Aboriginal people's health-care rights as identified in international law, constitutional law, and under the Treaties; respecting and meeting the distinct needs of individual Aboriginal communities; recognizing the value of Aboriginal healing practices and implementing them in practice and in collaboration with Aboriginal healers and Elders; increasing the numbers and retention of Aboriginal HCPs; and providing cultural competency training for all HCPs and healthcare students.

\section{Endnotes}

${ }^{1}$ In Canada, three types of Indigenous peoples (sometimes referred to as Aboriginal peoples) are constitutionally recognized: The First Nations, the Inuit, and the Métis peoples. Each group and each community is distinct in terms of their cultural identities, histories, languages, and so on. The Indigenous population represents approximately $4.3 \%$ of the total Canadian population.

${ }^{2}$ Residential schools were a federally funded policy whereby Indigenous children were removed from their homes, families, and culture, and forced into church-run schools aimed at assimilation into the dominant Canadian culture and society. The schools were in existence for over 100 years and have had a profound and continuing negative impact on Indigenous populations [34].

\section{Abbreviations}

CAC, community advisory committee; CBPR, community-based participatory research; HCP, healthcare provider

\section{Acknowledgments}

We would like to thank and acknowledge the communities of Maskwacis and the qualitative participants of the study for generating this knowledge with us. We would also like to acknowledge the assistance of Melissa Berg, Erinn Bailey-Sawatzky, Pattie Currie-Beebe, Sue Buffalo, Bonnie McCloud, Randy Littlechild, Gail Lightning, Joanne Siemens, Sarah Fordham, Gloria Mcknight, Maira Quintanilha, Hara Nikolopolous, Jessica Thompson, Jill Morris, Linda McCargar, Paula Robson, Sheila Tyminski, Dawn Phelps, Terri Miller, Dolly Bondarianzadeh, and Kelli Buckreus.

\section{Funding}

This study was funded by an Alberta Innovates - Health Solutions Collaborative Research and Innovation Opportunity team grant.

\section{Availability of data and materials}

The dataset supporting the conclusions of this article is not available, in order to preserve the anonymity of participants.

\section{Authors' contributions}

Oster contributed to the design, contributed to the data collection and analysis, and wrote the manuscript. Bruno contributed to the data collection and analysis. Montour/Roasting/Lightning/Rain/Graham were members of the Community Advisory Committee and as such contributed to the design and guided the project. Mayan/Toth/Bell contributed to the design and supervised the project. All authors contributed to manuscript writing, and approved the final manuscript.

\section{Authors' information}

Oster, PhD is a mixed-methods researcher with the Believing we can Reduce the Aboriginal Incidence of Diabetes research group at the University of Alberta, Department of Medicine. Bruno is a member of Samson Cree Nation and a research assistant for the ENRICH team at the University of Alberta, Department of Agricultural, Food and Nutritional Science. Montour is an Elder and cultural leader of Samson Cree Nation. Roasting is an Elder and cultural leader of Louis Bull Cree Nation. Lightning is an Elder, cultural leader, and previous member of Chief \& Council for Ermineskin Cree Nation. Rain is a member of Samson Cree Nation, and works for Maskwacis Health Services as the health manager for the Pigeon Lake Satellite Clinic. Graham, RN is the nurse manager for all of Maskwacis Health Services. Mayan, PhD is an associate professor and qualitative methodologist at the University of Alberta, Faculty of Extension. Toth, MD is a diabetes specialist with Maskwacis and Alberta Health Services, and a professor emeritus at the University of Alberta, Department of Medicine. Bell, PhD is a professor and human nutrition researcher at the University of Alberta, Department of Agricultural, Food and Nutritional Science.

\section{Competing interests}

The authors declare that they have no competing interests.

\section{Consent for publication}

Written informed consent to publish the findings of this research was obtained from the study participants.

\section{Ethics approval and consent to participate}

Ethical approval by the University of Alberta Research Ethics Board was obtained. Written informed consent to participate in the study was obtained from the study participants.

\section{Author details}

'Department of Medicine, Research Transition Facility, University of Alberta, Edmonton, AB T6G 2V2, Canada. 'Department of Agricultural, Food and Nutritional Science, Li Ka Shing Centre for Health Research Innovation, University of Alberta, Edmonton, AB T6G 2R3, Canada. ${ }^{3}$ Samson Cree Nation, Maskwacis, AB TOC 1NO, Canada. ${ }^{4}$ Louis Bull Cree Nation, Maskwacis, AB TOC 1NO, Canada. ${ }^{5}$ Ermineskin Cree Nation, Maskwacis, AB TOC 1NO, Canada. ${ }^{6}$ Maskwacis Health Services, Maskwacis, AB TOC 1NO, Canada. ${ }^{7}$ Faculty of Extension, Enterprise Square, University of Alberta, Edmonton, AB T5J 4P6, Canada.

Received: 25 May 2016 Accepted: 4 August 2016

Published online: 11 August 2016 


\section{References}

1. Calkins K, Devaskar SU. Fetal origins of adult disease. Curr Probl Pediatr Adolesc Health Care. 2011;41(6):158-76. doi:10.1016/j.cppeds.2011.01.001.

2. Shah PR, Zao J, Al-Wassia H, Shah V. Knowledge synthesis group on determinants of preterm/LBW births. Pregnancy and neonatal outcomes of aboriginal women: a systematic review and meta-analysis. Womens Health Issues. 2011;21(1):28-39. doi:10.1016/j.whi.2010.08.005.

3. Oster RT, King K, Morrish DW, Mayan MJ, Toth EL. Diabetes in pregnancy among First Nations women in Alberta, Canada: a retrospective analysis. BMC Pregnancy Childbirth. 2014;14(1):136. doi:10.1186/1471-2393-14-136.

4. Oster RT, Toth EL. Stillbirth epidemiology and risk factors among first nations and non-first nations pregnancies in Alberta, Canada from 2000 to 2009. J Obstet Gynaecol Can. 2015;37(2):117-21.

5. Statistics Canada. Aboriginal peoples in Canada in 2006, 2006 census: First Nations people. Ottawa: Statistics Canada; 2006.

6. Riddell CA, Hutcheon JA, Dahlgren LS. Differences in obstetric care among nulliparous first nations and non-first nations women in British Columbia. Can CMAJ. 2015;188(2):E36-43. doi:10.1503/cmaj.150223.

7. Oster RT, Mayan MJ, Toth EL. Diabetes in pregnancy among first nations women. Qual Health Res. 2014;24(11):1469-80. doi:10.1177/1049732314545089.

8. Sokoloski EH. Canadian First Nations women's beliefs about pregnancy and prenatal care. Can J Nurs Res. 1995;27(1):89-100.

9. Varcoe C, Brown H, Calam B, Harvey T, Tallio M. Help bring back the celebration of life: a community-based participatory study of rural aboriginal women's maternity experiences and outcomes. BMC Pregnancy Childbirth. 2013;13:26. doi:10.1186/1471-2393-13-26.

10. Wiebe AD, Barton S, Auger L, Pijl-Zieber E, Foster-Boucher C. Restoring the blessings of the morning star: childbirth and maternal-infant health for first nations near Edmonton. Alberta Aboriginal Policy Stud. 2015;5(1):47-68.

11. Heaman MI, Sword W, Elliott L, Moffatt M, Helewa ME, Morris H, et al. Perceptions of barriers, facilitators and motivators related to use of prenatal care: A qualitative descriptive study of inner-city women in Winnipeg. SAGE Open Med. 2015;3. doi: 3: 2050312115621314.

12. Tait NH. Patient and caregiver perspectives of health provision practices for first nations and Métis women with gestational diabetes mellitus accessing care in Winnipeg. Manit BMC Health Serv Res. 2014;14:440. doi:10.1186/ 1472-6963-14-440

13. Heaman MI, Sword W, Elliott L, Moffatt M, Helewa ME, Morris H, et al. Barriers and facilitators related to use of prenatal care by inner-city women: perceptions of health care providers. BMC Pregnancy Childbirth. 2015;15:2. doi:10.1186/s12884-015-0431-5.

14. Canadian Institutes of Health Research. CIHR Guidelines for Health Research Involving Aboriginal People. Ottawa: Health Canada; 2007.

15. Wallerstein N, Duran, B. The theoretical, historical, and practice roots of community-based participatory research. In Minkler M \& Wallerstein N (Eds.), Community-Based Participatory Research for Health: From Process to Outcomes. 2008. San Francisco, CA: John Wiley \& Sons. Chapter 2, p, 25-46.

16. Mayan MJ. Essentials of Qualitative Inquiry. Walnut Creek: Left Coast Press, Inc.; 2009.

17. Savage J. Ethnography in healthcare. BMJ. 2000;321(7273):1400-2. http://dx. doi.org/10.1136/bmj.321.7273.1400

18. Whittemore R, Chase SK, Mandle CL. Validity in qualitative research. Qual Health Res. 2001:11(4):522-37.

19. Milne J, Oberle K. Enhancing rigor in qualitative description: a case study. J Wound Ostomy Continence Nurs. 2005;32(6):413-20.

20. Beck RS, Daughtridge R, Sloane PD. Physician- patient communication in the primary care office: a systematic review. Am Board Fam Med. 2002;15:25-38.

21. Roter DL, Frankel RM, Hall JA, Sluyter D. The expression of emotion through nonverbal behavior in medical visits. Mechanisms and outcomes. J Gen Intern Med. 2006;21 Suppl 1:S28-34.

22. Doyle $C$, Lennox L, Bell D. A systematic review of evidence on the links between patient experience and clinical safety and effectiveness. BMJ Open. 2013:3(1):e001570. doi:10.1136/bmjopen-2012-001570.

23. Kurtz DLM, Nyberg JC, Van Den Tillaart S, Mills B. Okanagan urban aboriginal health research collective. Silencing of voice: an act of structural violence: urban aboriginal women speak out about their experiences with health care. J Aboriginal Health. 2008;4(1):53-63.

24. World Health Organization. WHO Recommendations on Health Promotion Interventions for Maternal and Newborn Health 2015. Geneva: World Health Organization; 2015.
25. Society of Obstetricians and Gynaecologists. Health professionals working with first nations, Inuit, and Métis consensus guideline. J Obstet Gynaecol Can. 2013;35 Suppl 2:S1-S52.

26. Birch J, Ruttan L, Muth T, Baydala L. Culturally competent care for aboriginal women: a case for culturally competent care for aboriginal women giving birth in hospital settings. J Aboriginal Health. 2009;4(2):24-34.

27. Couchie C, Sanderson S. A report on best practices for returning birth to rural and remote aboriginal communities. J Obstet Gynaecol Can. 2007; 29(3):250-60.

28. Skye A. Aboriginal midwifery: a model for change. J Aboriginal Health. 2010; 6(1):28-37.

29. Kildea S. Risky business: contested knowledge over safe birthing services for aboriginal women. Health Sociol Rev. 2006;15(4):387-96. doi:10.5172/hesr. 2006.15.4.387.

30. Van Wagner V, Osepchook C, Harney E, Crosbie C, Tulugak M. Remote midwifery in Nunavik, Québec, Canada: outcomes of perinatal care for the Inuulitsivik health centre, 2000-2007. Birth. 2012;39(3):230-7. doi:10.1111/j. 1523-536X.2012.00552.x.

31. Di Lallo S. Prenatal care through the eyes of Canadian aboriginal women. Nurs Womens Health. 2014;18:38-46.

32. Lu MC, Lin YG, Prietto NM, Garite TJ. Elimination of public funding of prenatal care for undocumented immigrants in California: a cost/benefit analysis. Am J Obstet Gynecol. 2000;182:233-9.

33. Jarvis C, Munoz M, Graves L, Stephenson R, D'Souza V, Jimenez V. Retrospective review of prenatal care and perinatal outcomes in a group of uninsured pregnant women. J Obstet Gynaecol Can. 2011:33:235-43.

34. Truth and Reconciliation Commission of Canada. Truth and Reconciliation Commission of Canada Calls to Action. 2015. Winnipeg, MN: Truth and Reconciliation Commission of Canada.

\section{Submit your next manuscript to BioMed Central and we will help you at every step:}

- We accept pre-submission inquiries

- Our selector tool helps you to find the most relevant journal

- We provide round the clock customer support

- Convenient online submission

- Thorough peer review

- Inclusion in PubMed and all major indexing services

- Maximum visibility for your research

Submit your manuscript at www.biomedcentral.com/submit
) Biomed Central 